\title{
Ce que m'a appris mon fils d'un an à propos de la pratique du pharmacien
}

par Jason Howorko

$\mathrm{L}^{\mathrm{a}}$ es selles: un phénomène qui se répète de 8 à 10 fois par jour... vraiment! Si j’ai personnellement appris quoi que ce soit au cours de cette dernière année, c'est bien à cause de l'arrivée de mon fils, Alexander. Tout en apprenant comment changer les couches, consoler un enfant en pleurs et ne pas pouffer de rire lorsque ma ravissante épouse est aspergée de nourriture, $\mathrm{j}$ 'ai aussi beaucoup appris sur notre profession.

Faites-vous des amis : Dans notre profession, nous devons nous serrer les coudes. Rencontrez vos collègues et d'autres professionnels de la pharmacie de votre service, de votre hôpital, de votre ville, de votre province, de votre association professionnelle et de votre pays. Ensemble, nous pouvons nous faire entendre.

N'oubliez pas de partager : Bien que de voir Alex « voler " le petit camion de son ami Rylee puisse être quelques fois tout un spectacle, cela me rappelle que nous avons tous besoin de ne pas oublier de partager. Chacun de nous fait des choses formidables dans sa pratique individuelle. N'oubliez pas de les partager avec l'univers au moyen d'articles, de présentation par affiches, de courriels dans les Réseaux de spécialistes en pharmacie!

Profitez des petites choses : Malgré que mon fils ait dit le mot « $\mathrm{PaPa}$ " pour la première fois alors qu'il poursuivait le chat, j'étais quand même tout fier. N'oubliez pas de profiter des petites choses que nous faisons et de savourer les petits succès que nous avons.

Allez-y petit à petit vers de grandes réalisations : Je me souviens des premiers pas d'Alex. Dans les semaines qui ont suivi, il vacillait et tombait chaque fois qu'il essayait de marcher, mais il ne s'est jamais découragé. Maintenant qu’il court partout, il me rappelle que nous devons y aller petit à petit pour atteindre nos objectifs ultimes.

Amusez-vous : De temps à autre, nous devons tous prendre un peu de recul et nous amuser. Bien que de jouer à coucou avec un collègue sur l'unité de soins puisse à tout le moins susciter l'étonnement, on peut tout au moins envisager de participer à une conférence de la Société canadienne des pharmaciens d'hôpitaux (SCPH). Une façon d'apprendre en s'amusant!
Sachez vous adapter : Tout comme l'enfant grandit et se développe, il en est ainsi de notre profession. Chaque jour, l'enfant acquiert de nouvelles habiletés et repousse les limites de son monde. Le jour où Alex rampant au sol a trouvé le moyen de s'emparer de la télécommande sur le canapé en tirant le coussin à lui, je savais que ma vie ne serait plus jamais la même... l'élève de neuf mois avait dépassé le maître! Le changement peut arriver rapidement et parfois il faut savoir s'y conformer.

Malgré que nous ne puissions pas tout prévoir, nous pouvons et nous devons nous adapter rapidement face aux nouvelles occasions et aux nouveaux défis qui se présentent. Nous devons bâtir des liens, grandir, apprendre, nous développer, nous adapter, partager, faire quelques petits pas, tomber, faire quelques pas de géant, savourer les petites choses et ne pas oublier de nous amuser.

Quand je regarde dans les yeux de mon fils, je rêve pour lui d'un monde rempli d'occasions. Aujourd'hui, je vois aussi des occasions pour notre profession qui sont à portée de main. Nous pouvons faire toute la différence, une Société, une section provinciale, un service de pharmacie et un pharmacien à la fois. Alors, je ne peux qu'imaginer où nous serons rendus dans dix ans.

J'ai eu l'immense plaisir de servir la SCPH à titre de délégué présidentiel ces trois dernières années. Je chérirai à jamais ces moments précieux et les nouvelles amitiés qui se sont forgées dans le cadre de mon mandat, tout comme j'affectionne l'expérience de la paternité. Salutations!

[Traduction par l'éditeur]

Jason Howorko, BSP, B. Sc., ACPR, est Président sortant et agent de liaison externe de la SCPH. 